\title{
The Maxwellian optics laboratory automation using a personal computer
}

\author{
NAOYUKI OSAKA \\ Otemon-Gakuin University, Ibaraki, Osaka, Japan
}

\begin{abstract}
The Maxwellian optical system described here is fully automated in both experimental control for psychophysical instrumentation and data gathering using a personal computer (PC) with simple interfacing and input/output processing. The system features PC-based high-speed noise-free liquidcrystal shutter control, automated light control, glow modulator tube control, and filter/wedge control. PC-based data gathering and data transfer to the mainframe via RS-232C-based Telocom are described.
\end{abstract}

The Maxwellian-view optical system for presenting a visual stimulus is an apparatus by which an observer can increase the quantity of achromatic/monochromatic light reaching the retina of an observer. The system, of course, is widely used in vision research laboratories (see Westheimer, 1966).

Problems involved in using the classical Maxwellian optics include noise from mechanical shutter operation, difficulty of precise light control, and manual operation of stimulus pattern change. A noise-free and high-speed shutter is important for visual experiments testing the effects of flash duration and subsequent visual evoked potentials (VEP) (see Osaka, 1984). A personal-computer (PC)based light control and automated stimulus changer is critical for experimental control.

A PC-controlled noise-free liquid-crystal shutter (LCS) is one solution to the problem of noise and speed of response (maximum of approximately $5 \mathrm{msec}$ ), since a LCS has no mechanical structure. A PC-based optical system can also solve some of the remaining problems. Control of the duration of pulsed light, light control by neutral density filter/wedge, luminance calibration, wavelength control, and data acquisition with the Maxwellian optics appear relatively easy and inexpensive using low-cost PCs.

A low-cost PC (e.g., Commodore's PET/CBM, VIC20, NEC's PC 9801,8801 , or Fujitsu's FM-8, FM-11 PCs) provides a relatively inexpensive means of controlling vision experiments (Osaka, 1983b) and data acquisition/analysis, and even serves as a Telecom terminal emulator (Osaka, 1983a). A diagram of the Maxwellian optical system is shown in Figure 1. Any kind of PC incorporating a bit-addressable parallel input/output port, general-purpose interface bus (GPIB), and RS-

This work was supported by Grants 58510069 and 59510073 from the Ministry of Education, Japan. For reprints, write to N. Osaka, Department of Psychology, Otemon-Gakuin University, Ibaraki, Osaka 567 , Japan.

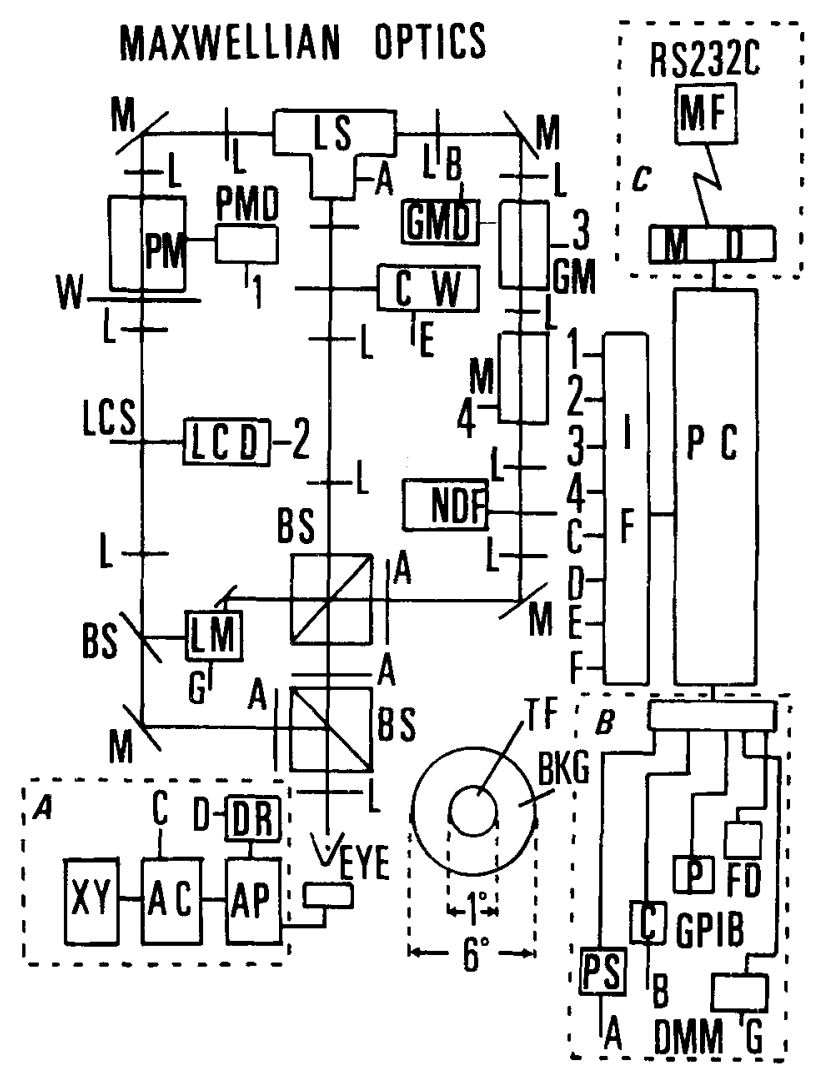

Figure 1. PC-controlled Maxwellian optical system. LS: light source; GM: glow modulator tube (R1131C); GMD: glow modulator driver; L: lens; M: mirror; PM: pulse motor; PMD: pulse motor driver; PS: power supply; C: counter; P: printer; FD: floppy disk; W: wheel; MF: mainframe computer; LCS: liquid-crystal shutter; LCD: liquid-crystal driver; BS: beam splitter; A: aperture; CW: circular wedge; M: monochromator; NDF: neutral density filter; DR: data recorder; AC: averaging computer (ATAC-450); PC: personal computer; AP: amplifier; XY: X-Y plotter; MD: modem; DMM: digital multimeter; LM: luminance meter; TF: test field; BKG: background view. The dashed areas $A, B$, and $C$ indicate VEP-, GPIB-, and RS-232C-laboratory, respectively. 
232C serial communication interface is applicable for the system controller PC.

\section{A BIT-ADDRESSABLE I/O PORT}

A one-chip programmable integrated large-scale circuit (LSI) makes design of psychophysical instrumentation, such as Maxwellian optics, simple and inexpensive for real-time research. One can easily build an intelligent parallel communication interface with little electronics knowledge by using a one-chip peripheral versatile interface adaptor (VIA) coupled with a one-chip Darlington switch (Osaka, 1981). Furthermore, a researcher can easily control the $\mathrm{I} / 0$ ports by simply putting a bit pattern on the control registers. Programming for $\mathrm{I} / \mathrm{O}$ processing is also simple by using PEEKing/POKEing at each I/O register address (Osaka, 1979a, 1979b, 1980).

This type of a bit-addressable I/O register controls the LCS (Nitten Corporation), pulse motor (PM; Nippon Pulse Motor Corporation, PA62), glow modulator (GM) tybe (Sylvania Corporation, R1131C), monochromator (M; Jobin-Yvon, H20 with $\mathrm{I} / \mathrm{O}$ slit width of $1 \mathrm{~mm}$ ), and circular neutral density wedge (CW; Kodak, A6020).

The LCS can be activated by an LC driver (LCD; $99 \mathrm{~V} \mathrm{dc}$ ) with a TTL-level trigger pulse input: The duration of the shutter operation can be controlled by the pulse width given by the PC. Figure 2 shows an example program for LCS control. The LCS has rise and fall times shorter than $4.5 \mathrm{msec}$ which enables high-speed shutter control without mechanical noise. GM tube control can be achieved by providing a pulse on the trigger gate of the GM tube driver (GMD) (Osaka, 1979a). Furthermore, the monochromator $(\mathrm{M})$ and the stimulus wheel (see Osaka, 1979a) connected to the associated pulse motor can be controlled by supplying a pulse to the pulse motor driver (PMD): A pulse rotates the associated axis of the monochromator (M) for a $6^{\circ}$ step angle. Similarly, a pulse rotates the pulse motor for a $15^{\circ}$ step angle; thus, 24 pulses result in one rotation $\left(360^{\circ}\right)$ of an associated stimulus wheel (Osaka, 1979a).

A bit-addressable $\mathrm{I} / \mathrm{O}$ port also controls the averaging computer (AC; Nihon Kohden Corporation, ATAC-450) and data recorder (TEAC Corporation, R250).

\section{GPIB-BASED SUBSYSTEM}

Spot luminance meter (Minolta, $1^{\circ}$ digital) continuously measures the light output in order to maintain the amount of light at a constant luminance level that is predetermined and stored in the PC program (Osaka, 1982). The PC reads the analog-to-digital converted luminance value from the GPIB-driven digital multimeter (DMM; Takeda Riken Corporation, TR6841) connected to the luminance meter and compares it with a predetermined level. If the difference between the previously stored and the measured value is greater (less) than some permissible value, say, $2 \mathrm{~cd} / \mathrm{m}^{2}$, the $P C$ adjusts the luminance level by rotating the $\mathrm{CW}$ until both values coincide. This feedback looping can be done by a BASIC program using a conditional "IF ... THEN" statement (Figure 2). This looping has merit in detecting a small deviation of the light. Figure 2 shows an example program for light control. Our simple GPIB-based laboratory system includes (Fisher \& Jensen, 1980; Osaka, 1983b): The power supply (PS; Takasago Corporation, TC801), digital counter (C; Takeda Riken Corporation, TR5822), tractor printer (P; Commodore Corporation, CBM3022), and floppy disk (FD; Commodore Corporation, CBM3040), all connected to the PC. (Refer to Figure 1, dotted line area B, at the lower right portion of the figure.)

\section{VEP LABORATORY SECTION}

The VEP averaging processor (AC; Nihon Kohden Corporation, ATAC-450 system) receives an amplified electroencephalogram from the observer's electrodes at oc-

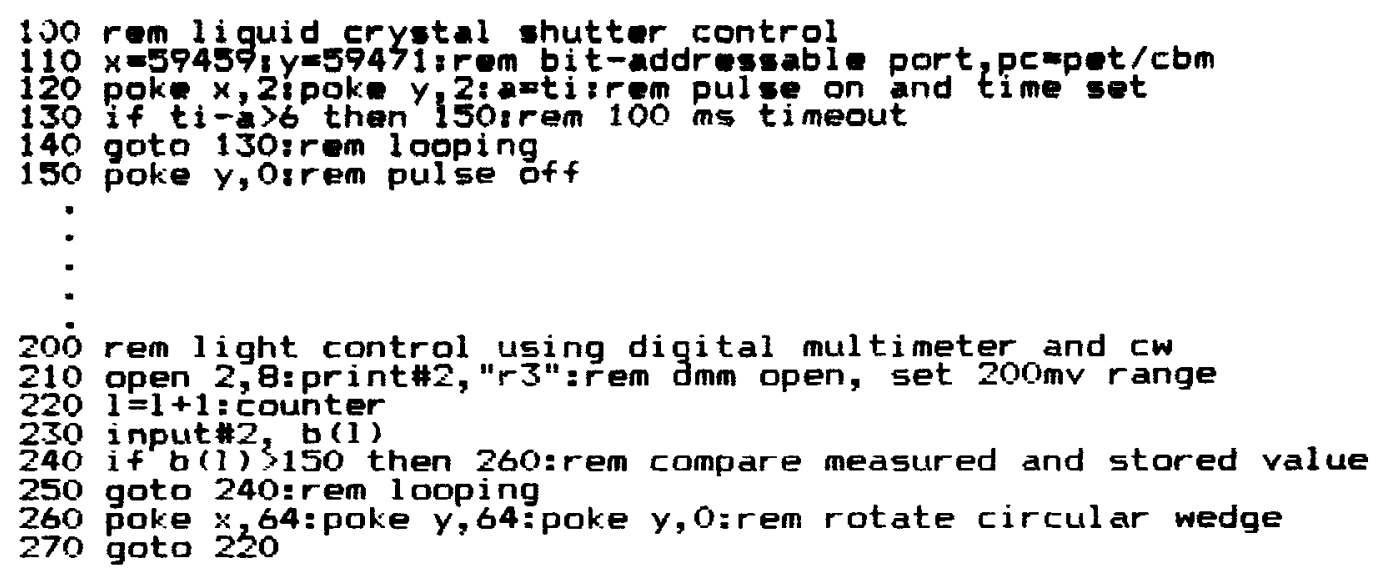

Figure 2. Sample BASIC program for LCS and light control. 
cipital positions (dotted-line area A in Figure 1). After 10 min of dark adaptation, the observer is asked to fixate the center of the test field (TF): When the PC triggers the LCS and AC simultaneously, a brief flash without noise appears on the TF, which evokes a visual evoked potential. VEP averaging and its power spectra can be estimated using a Fast Fourier Transformation (FFT) algorithm (Osaka, 1984).

\section{RS-232C-BASED SUBSYSTEM}

The RS-232C-based communication system is shown in Figure 1 (upper right, dotted-line area $\mathrm{C}$ ). The $\mathrm{PC}$ with RS-232C interface capability transfers gathered data to the mainframe computer (MF; Fujitsu M382) via an acoustic coupler (MD; Tamura Corporation, ACTAM360A1). The data gathered during an experiment is stored on floppy disk (FD) and then transferred to the host mainframe in the Data Processing Center, Kyoto University, via TTYprotocols [i.e., 7-bit data length, 1-stop bit, even parity, and $300 \mathrm{bps}$ half-duplex mode (Osaka, 1983a)]. Usually we use Statistical Analysis System (SAS) and SAS/ GRAPH statistical packages for further data analysis at the host computer.

\section{SUMMARY}

The LCS works quite well with the PC; however, there appears to be a slight loss in light intensity due to the absorption within a liquid crystal when a beam passes through it. Light control, as well as pulse motor control, works well with the PC.

\section{REFERENCES}

Fisher, E., \& JENSEN, C. N. (1980). PET and the IEEE-488 bus (GPIB). Berkeley, CA: Osborne/McGraw-Hill.

OSAKA, N. (1979a). A microprocessor-based real-time BASIC laboratory: A pulse motor-controlled visual stimulator. Behavior Research Methods \& Instrumentation, 11, 549-552.

OSAKA, N. (1979b). A microprocessor-based real-time BASIC random interstimulus interval generator with different probability density functions. Behavior Research Methods \& Instrumentation, 11, 581-584.

OsAKA, N. (1980). An inexpensive microcomputer peripheral I/O expansion. Behavior Research Methods \& Instrumentation, 12, 344-345.

OSAKA, N. (1981). A versatile memory-mapped I/O interface for microcomputers. Behavior Research Methods \& Instrumentation, 13, 727-731

Osaka, N. (1982). Exponent of the Broca-Sulzer flash duration as a function of retinal eccentricity. Journal of the Optical Society of America, 72, 62-67.

OSAKA, N. (1983a). [Computer communication: An instrumentation for psychological experiment and communication]. Kyoto: Nakanishiya Publishing. (In Japanese)

Osaka, N. (1983b). [Computer control: An instrumentation for psychological experiment and communication]. Kyoto: Nakanishiya Publishing. (In Japanese)

OSAKA, N. (1984, October). The Broca-Sulzer visual evoked potentials: Brightness- and darkness-versions. Paper presented at the annual meeting of the Optical Society of America, San Diego, CA.

Westhermer, G. (1966). The Maxwellian view. Vision Research, 6, 669-682. 